\title{
Estresse infantil e sua relação com o rendimento escolar da criança nas disciplinas de português e matemática
}

\section{Child stress and its relationship with childhood performance in portuguese disciplines and mathematics}

Rafael Ayres Romanholo ${ }^{1,2}$, Wesley Welington Garbin Theodoro ${ }^{3}$, Fernando Costa Baia ${ }^{4}$, Joeliton Elias Pererira ${ }^{5}$, Adriano Robson Nogueira da Lucena ${ }^{6}$, Jonato Prestes ${ }^{7}$, Fabricio Moraes de Almeida ${ }^{8}$

1. Doutorando em Desenvolvimento Regional e Meio Ambiente pela Universidade Federal de Rondônia (UNIR), Porto Velho, RO, Brasil. 2. Docente do Instituto Federal de Rondônia (IFRO), Cacoal, RO, Brasil. 3. Discente do curso de Educação Física pela Faculdade de Ciências Biomédicas de Cacoal (FACIMED), Cacoal, RO, Brasil. 4. Pesquisador do Grupo de Pesquisa em Motricidade Humana, Sociedade e Saúde (GPMOSOS), do Instituto Federal de Rondônia (IFRO), Cacoal, RO, Brasil. 5. Docente da Faculdade de Ciências Biomédicas de Cacoal (FACIMED), Cacoal, R0, Brasil. 6. Pesquisador do Grupo de estudos do Laboratório de Biociências do Movimento Humano (LABIMH), da Universidade Federal do Rio de Janeiro (UFRJ), Rio de Janeiro, RJ, Brasil. 7. Professor da Universidade Católica de Brasília. UNB. 8. Docente do Programa de Pós-graduação em Desenvolvimento Regional e Meio Ambiente pela Universidade Federal de Rondônia (UNIR), Porto Velho, RO, Brasil.

\section{Resumo}

Introdução: Nota-se que o estresse influencia no rendimento escolar, e este é influenciado por diversos fatores. Objetivo: Verificar o rendimento escolar e o estresse em escolares de 7 a 12 anos no município de Cacoal/RO. Método: As crianças responderam aos protocolos de Índice de stress infantil - ISSI-I, sendo um questionário com 35 questões, que relacionavam as dimensões do estresse infantil, psicológico ou físico, com o rendimento escolar, disponibilizado pela escola, cujo resultado correspondia às médias dos alunos em Português e Matemática dos quatro bimestres, os quais foram selecionados para a comparação e a análise dos resultados. A amostra do estudo contou com 230 crianças do sexo masculino e 252 crianças do sexo feminino. Resultado: 0 estudo mostrou relação entre escolares que estão sob condições de stress e seu rendimento escolar. Ainda é importante verificar se os vários sintomas estão ocorrendo juntos. Conclusão: 0 stress não tratado e prolongado pode levar a uma série de doenças e problemas de adaptação, inclusive na escola.

Palavras-chave: Estresse. Educação. Infantil. Redimento Escolar

\begin{abstract}
Introduction: It is noted that stress influences school performance, and this is influenced by several factors. Objective: This study aimed to verify school performance and stress in schoolchildren aged 7 to 12 years in the municipality of Cacoal / RO. Methods: The children responded to the ISSI-I protocols, with a questionnaire with 35 questions relating the dimensions of the child's psychological or physical stress to the school's performance of the averages (Mathematics and Portuguese) of the four two-month periods of the students selected to compare and analyze the results. The study sample consisted of 230 males and 252 females. Results: The study showed the number of schoolchildren who are over stress and the relationship with their school performance. It is still important to check if various symptoms are occurring together. Conclusion: Untreated and prolonged stress can lead to a number of diseases and problems of adjustment, including at school.
\end{abstract}

Key words: Stress. Education. Infantile. Underachievement

\section{INTRODUÇÃO}

As interações do ser humano e dos animais com o meio requerem respostas psicológicas e comportamentais perante os constantes desafios que lhes são impostos1. Para Lemes (2003), o estresse é visto como parte do sistema biopsicossocial adaptativo. Isso mostra que, para o ser humano ser capaz de responder a essas demandas, é necessário superar adversidades impostas pelo meio, muitas vezes, geradoras de desequilíbrio, tanto em termos físicos como psicológicos.

Esse equilíbrio é denominado homeostase e corresponde à capacidade de autorregulação dos vários sistemas existentes no organismo, de modo que, em face das alterações no equilíbrio interno, o indivíduo reage de forma para buscar estabelecer posição similar à que existia previamente à ocorrência do desequilíbrio ${ }^{1}$.

Em 1936, Hans Selye, um dos precursores dos estudos sobre o estresse, definiu-o como "o resultado inespecífico de qualquer demanda sobre o corpo, seja de efeito mental ou somático"; e estressor, como todo agente ou demanda que evoca reação de estresse, seja de natureza física, mental ou emocional ${ }^{2,3}$. O conceito de estresse foi, então, formulado em seus trabalhos com animais de laboratório. Selye percebeu que, quando esses animais eram submetidos a situações hostis, em que se excediam suas capacidades de defesa, apresentavam uma série de alterações fisiológicas ${ }^{1,4}$.

O estresse é parte do sistema biopsicossocial adaptativo. $\mathrm{Na}$ interação do ser humano e dos animais com o meio, são requeridas respostas psicológicas e comportamentais diante dos constantes desafios que lhes são impostos ${ }^{5}$. 0 termo stress foi definido, do ponto de vista fisiológico, por Selye (1965) na década de 1930 e trouxe como grande contribuição a descrição

Correspondência: Rafael Ayres Romanholo. Universidade Federal de Rondônia (UNIR), Porto Velho, RO, Brasil. Av. Pres. Dutra, 2965 - Centro, Porto Velho - R0, 76801-974. E-mail: rafael.ayres@ifro.edu.br 
de uma resposta não específica a qualquer demanda que se imponha ao corpo. Esta resposta pode influenciar a recuperação de doenças, reduzir a resistência a elas ou até produzi-la.

Para Lipp et al., ${ }^{6}$ o stress infantil é semelhante ao do adulto; a criança quando se vê diante de um estressor reage com sensações físicas e psicológicas, sendo que as consequências do stress excessivo ou prolongado podem levar a problemas graves de saúde. Ainda os estudos apontam que é na escola onde aparecem os principais sintomas de estresse.

A escola é vista como um contexto que privilegia as interações da criança com seus pares, em decorrência do número de horas diárias que elas passam nesse ambiente. Porém, é possível verificar que uma dessas formas de interação pode ser o conflito, considerado como uma oposição comportamental.

Com base nesse pressuposto, é possível verificar que o conflito entre pares no contexto escolar surge como um estressor específico muito comum entre as crianças das séries iniciais. Isso indica que a escola, considerada como o primeiro ambiente socializante para a criança fora do seio familiar, pode tornar-se uma fonte de estresse, conforme Lipp et al., ${ }^{6}$. Leme ${ }^{1}$ explicita ainda que tanto o professor como o aluno trazem para o ambiente escolar seu histórico de vida, suas ideias, concepções e expectativas mútuas do que deverá ocorrer em sala de aula.

Com isso, o objetivo da pesquisa foi analisar a relação entre o estresse infantil e o rendimento escolar de indivíduos de 5 as 12 anos no Município de Cacoal/RO.

\section{MATERIAL E MÉTODOS}

Para o estudo com escolares, a amostra foi selecionada por meio de uma demonstração probabilística estratificada, que foi retirada de 220 alunos, com a idade de 5 a 12 anos, do sexo masculino e feminino. Para a seleção da amostra, foi aplicado o cálculo estatístico de Kazmier ${ }^{7}$, no qual foi respeitada uma margem de erro de $0,5 \%$ e um percentual de $8,7 \%$ da população total de escolares dessa faixa etária e gênero, distribuídos em seis escolas no município de Cacoal/RO.

As escolas foram distribuídas em setores e, com o auxílio do mapa do município, verificamos a distribuição das escolas. Utilizamos uma escola de cada região (nas regiões em que havia mais de uma escola, foi feito um sorteio simples para a escolha daquela que seria avaliada), para obtermos dados significativamente distribuídos no município. Como critérios de inclusão, foram adotados: os pais assinarem o Termo de Consentimento Livre e Esclarecido; o escolar ter nascido no estado de Rondônia; estar devidamente matriculado no ensino público e privado no município de Cacoal e ter idades entre 5 a 12 anos, além de não apresentarem deficiências físicas, motoras e neurológicas.

Este estudo é classificado como transversal e tem características descritivas com um perfil de pesquisa Quali-quantitativo, em que, além de quantificar os dados e utilizar cálculos estatísticos para sua realização, também irá classificar os grupos enquanto características populacionais. Para a avaliação dos agentes considerados estressores pela criança, utilizou-se a Escala de Stress Infantil (ESI) de Lipp e Lucarelli ${ }^{8}$ e Lipp e Malagris ${ }^{9}$, a qual permite diagnosticar se a criança apresenta um Quadro sintomatológico de estresse em níveis de Alerta, Resistência, Quase-Exaustão e Exaustão.

O presente instrumento é composto de um manual e um caderno de aplicação, que contém 35 afirmações, agrupadas em reações físicas, psicológicas, psicológicas com componente depressivo e reações psicofisiológicas, apresentando uma limitação, já que ele não é validado para a idade de 5 anos. Além disso, um protocolo de interpretação, no qual a criança faz registros, colorindo o quarto de círculos posicionados ao lado das afirmações. A apuração dos resultados foi feita por meio da contagem de pontos atribuídos à escala Likert, sendo que cada quarto de círculo equivale a um ponto. Como podemos observar nos exemplos:

Pode-se dizer que a criança avaliada tem sinais significativos de stress, quando: aparecerem círculos completamente cheios (pintados) em sete ou mais itens da escala total, ou obtiver nota igual ou maior que 27 pontos em qualquer dos três fatores a seguir: reações físicas (itens 2,6,12,15,17,19,21,24 e 34); reações psicológicas (itens 4,5,7,8,10,11,26,30 e 31); e reações psicológicas com componente depressivo (itens $13,14,20,22,25,28,29,32$ e 35 ) ou nota igual ou maior que 24 pontos obtida no fator reações psicofisiológicas (itens 1,3,9,16,18,23,27 e 33), ou nota total da escala maior que 105 pontos.

O estresse para Lipp ${ }^{11}$ possui modelo quadrifásico, composto por fase de alerta, fase de resistência, fase de quase exaustão e fase de exaustão. Na fase inicial do estresse, é produzida a adrenalina que gera dificuldade de dormir, produtividade, criatividade, respiração e humor eufórico. Se o esforço for suficiente para lidar com a situação, o estresse será eliminado, e a pessoa será capaz de superar o processo de estresse. No presente estudo, as crianças foram separadas em turmas de 15 escolares de ambos os sexos, sendo selecionada uma sala de aula para a aplicação do inventário. Para aplicação do Teste 3, acadêmicos do curso de Bacharelado em Psicologia auxiliaram os pesquisadores na aplicação do Inventário, em que as crianças eram dirigidas para uma sala de aula com ele, levando também lápis e borracha. Os acadêmicos liam cada item para as crianças, explicando o procedimento até o preenchimento total do inventário.

Para a coleta do rendimento escolar, foi utilizada a média do primeiro semestre de 2013 das disciplinas de Português e Matemática, obtida por meio dos boletins cedidos pelas secretarias das escolas. Para a análise da normalidade amostral, foi utilizado o teste de Kolmogorov-Smirnov e utilizado o programa SPSS 17,0 . Por meio das medidas de tendências central, média e desvio padrão, fizemos a caracterização das variáveis medidas e das contínuas escalas medidas. Para comparar os dados, foi utilizado o teste de Anova. Para 
determinar as variáveis a integrar o modelo de regressão linear, foi inicialmente aplicado o teste de correlação de Pearson $(r)$ para verificar a associação entre as variáveis.

A pesquisa foi autorizada pelo CEP-FACIMED/RO com o número de protocolo 00611/11. 1.530.602.

\section{RESULTADOS}

A tabela 1 apresenta os valores descritivos das variáveis Estresse e Rendimento escolar. $O$ estresse se enquadrou dentro do perfil de resistência em que o valor ficou na média 6.3 da escala de stress. Quando verificada a idade cronológica das amostras, pôde-se verificar que a média ficou em 8,7 anos e d.p +- 1,9 anos. Quando avaliado o rendimento escolar por meio das notas, pôde-se verificar que, na disciplina de Língua Portuguesa, as crianças alcançaram a média de 75 e d.p +- 8,34, e na disciplina de Matemática, as crianças alcançaram a nota de 72 d.p +- 9,76, como pode ser visto na tabela abaixo:

Tabela 1. Análise descritiva das variáveis: Idade cronológica, Stress e rendimento escolar (Português e matemática).

\begin{tabular}{lrrr}
\hline \multicolumn{1}{c}{ Variáveis } & No & Média* & Dp \\
\hline Idade Cronologia & 220 & 8,7 & 1,9 \\
Stress & 220 & 6,3 & 1,2 \\
Notas de Português & 220 & 75 & 8,34 \\
Notas de matemática & 220 & 72 & 9,76 \\
\hline *Anos & & &
\end{tabular}

A tabela 2 mostra as relações entre estresse e nota de Português e estresse e nota de Matemática. Pode-se perceber que houve relação em todas as variáveis analisadas, como pode ser visto na tabela abaixo.

Tabela 2. Relação entre as variáveis Stress e Rendimento escolar.

\begin{tabular}{lrrr}
\hline Variáveis & No & $\mathbf{r}$ & $\mathbf{p}$ \\
\hline Stress $\times$ N.P & 220 & 0,32 & $0,374^{*}$ \\
Stress $\times$ N.M & 220 & 0,21 & $0,467^{*}$ \\
\hline
\end{tabular}

$\mathrm{N} . \mathrm{P}=$ Notas de português/ N.M= Notas de matemática $/{ }^{*}$ significância

A tabela 3 mostra a análise de regressão linear entre a variável dependente Stress e a variável independente Rendimento escolar: notas de matemática e Português. Pode-se verificar que, nesta regressão, as variáveis independentes interagiram com o estresse com $65 \%$ de interferência e um $p=0.000$.

Tabela 3. Analise de regressão linear das variáveis Stress e notas de matemática e português analisadas no estudo

\begin{tabular}{llllll}
\hline Variáveis & $\boldsymbol{\beta}$ & $\mathbf{t}$ & sig & ?2 & sig \\
\hline Português & 0,181 & 3,301 & $0,001^{*}$ & & \\
Matemática & 0,132 & 2,658 & $0,008^{*}$ & 0,65 & $0,000^{*}$ \\
\hline
\end{tabular}

* significância

J. Health Biol Sci. 2017; 5(2):155-159

\section{DISCUSSÃO}

Pesquisas desenvolvidas por Cause e Dubow ${ }^{10}$ e Compas et al. ${ }^{11}$ evidenciaram que o estudo de estressores específicos é considerado um ganho em decorrência do uso de diferentes estratégias para estressores distintos, se for considerado que os fatores do meio, as variáveis pessoais, em especial as avaliações de ameaça e o controle sobre o estressor, ou as exigências da situação estressante poderão gerar influências ${ }^{12}$.

Para Lipp et al., ${ }^{13}$, as exigências impostas pelo contexto escolar, durante seu desenvolvimento, exigências em que a criança se vê obrigada a adaptar-se, podem ser geradoras de estresse. Contudo, a sintomatologia do estresse não afeta a todas as crianças de maneira uniforme, visto que cada pessoa interpreta os eventos de uma maneira, podendo eles ser vistos como estressores ou não. Por isso, deve haver a relevância da busca por mecanismos defensivos, psicologicamente adaptativos, bem como a prevenção da instalação dos sintomas, visando à proteção a essas crianças.

Torna-se necessário, dessa forma, inicialmente, a identificação de condições que poderiam contribuir para o desenvolvimento do estresse em crianças, em decorrência da escassez de estudos a respeito do estresse infantil.

Em 1980, Grunspun já fazia inferências sobre a existência do estresse no infante, porém, sem respaldo científico considerável para mensurá-lo e diagnosticá-lo. Em 1987, Lipp e Romano publicaram uma versão preliminar de um Inventário de Sintomas de Estresse, o qual veio a ser validado por Lucarelli em 1997 e deu origem à Escala de Estresse Infantil, viabilizando os estudos a respeito do levantamento do estresse em crianças $^{9,10}$.

Vilela ${ }^{14}$ comparou o nível de estresse em crianças do Ensino Fundamental de escolas particulares e públicas. Neste estudo, foi verificado que as crianças apresentavam o estado de atenção dentro do Inventário, corroborando os achados no atual estudo, em que as crianças analisadas também estão dentro da classificação de atenção.

Tricoli $^{15}$ averiguou a sintomatologia em escolares, e o que mais o chamou a atenção foi o sintoma de dor de cabeça e dor de "barriga". No atual estudo, o sintoma que mais chamou a atenção foi a dor de cabeça seguida por dor abdominal. Observa-se que os dois estudos apontam a dor de cabeça como um sintoma que requer a atenção de pais e professores. No estudo de Pereira16, analisou-se a correlação entre o estresse e a dor abdominal sem causa física em 46 crianças de 7 a 14 anos; porém, nota-se que a dor de cabeça aparece no estudo como o terceiro fator de sintomatologia que pode corroborar $o$ atual estudo.

Ainda que exista a necessidade de estudos a respeito da sintomatologia do estresse infantil, é possível identificar os sintomas de estresse mais prevalentes em crianças, entre eles: aparecimento súbito de comportamentos agressivos, 
não representativos do comportamento do infante no geral; desobediência inusitada; dificuldade de concentração, sintomas de depressão, ansiedade, enurese, gagueira, dificuldades de relacionamento, dificuldades escolares, pesadelos, insônia, birras e até o uso indevido de tóxicos.

Além disso, há ocorrência de causas físicas relacionadas ao estresse, como asma, bronquite, hiperatividade motora, doenças dermatológicas, úlceras, obesidade, cáries, cefaleia, dores abdominais, diarreia, tiques nervosos, entre outros. Em estudo realizado por Rodrigues et al. ${ }^{17}$, os pesquisadores avaliaram o rendimento escolar em crianças intoxicadas por chumbo. Segundo o estudo, os dados confirmam os apontamentos sobre prejuízos à saúde e ao desenvolvi-mento infantil, indicando relações entre a contaminação por chumbo e as dificuldades de aprendizagem. Nota-se que, no atual estudo, muitas crianças são da zona rural, onde o uso de agrotóxicos é alto nas plantações, e a matéria prima desses agrotóxicos são os metais pesados. Isso pode ser um agravante que potencializou os sintomas do estudo de Rodrigues e o atual.

A grande dificuldade em associar essas variáveis é citada por Lipp ${ }^{9}$, em que os sintomas de estresse infantil não são sempre diagnosticados. Desse modo, pais e professores acabam por punir e coagir a criança em decorrência de mudanças súbitas de comportamento ou queda do rendimento escolar. Essas atitudes dos adultos diante de situações como estas tendem a agravar o problema, pois se torna mais uma fonte de estresse para a criança, visto que ainda não compreende o que se passa em seu organismo.

De acordo com Lipp ${ }^{18}$, a incidência de estresse grave na população infantil não é conhecida, porém, há conhecimento a respeito das queixas que são responsáveis pelo desenvolvimento da sintomatologia do estresse nessa população. Esse pressuposto embasa a referência às queixas do infante em relação aos aversivos geradores de estresse no contexto escolar, entre os quais, está a irritabilidade demonstrada pelo professor, os gritos, a impaciência e a falta de comunicação com os alunos, as instruções confusas, a escassez de elogios, o desconhecimento dos direitos do alunado, não ser compreensivo quando a criança queixa-se sobre a existência de algum conflito no contexto familiar, o excesso de tarefas, a manutenção de comportamentos de competição entre os colegas de turma e a desorganização. Isso mostra o quanto a relação entre a criança e o professor pode contribuir para o desenvolvimento do estresse infantil.

O ingresso no contexto escolar pode ainda influenciar e dificultar o desenvolvimento intelectual, emocional e afetivo da criança, visto que todas essas etapas trazem consigo também inúmeras situações geradoras de tensão, muitas vezes incapacitantes para as crianças e para o seu ainda frágil mecanismo de combate ao estresse. Isso é apontado por Tricoli ${ }^{19}$, o qual explana que, atualmente, com a necessidade de as mães entrarem no mercado de trabalho, as crianças têm iniciado a vida escolar cada vez mais precoce.

As exigências impostas pelo contexto escolar durante o seu desenvolvimento humano, exigências em que a criança se vê obrigada a adaptar-se, podem ser geradoras de estresse. Contudo, a sintomatologia do estresse não afeta todas as crianças de maneira uniforme, visto que cada pessoa interpreta os eventos de uma maneira, podendo este ser visto como estressor ou não. Com isso, cabe a pais, professores e família ficar atento à sintomatologia do estresse para que uma possível aresta na criança não se torne um grande problema no adulto.

\section{CONCLUSÃO}

Comparando esses achados com o atual estudo, pode-se verificar que há uma tendência em associar o estresse ao rendimento escolar, visto que as crianças avaliadas apresentavam-se em estado de atenção e com as notas no limite de suas médias.

No atual estudo, pôde-se verificar que as crianças apresentavam sintomas de stress, porém, não foram avaliados possíveis agentes estressores. No inventário de stress, o que mais chamou a atenção foram as respostas de sintomas físicos, como dor de cabeça e dor de barriga. Sugere-se que haja um aprofundamento longitudinal nos dados achados, em que poderão ser desenvolvidas pesquisas que acompanhem a evolução dessas crianças na escola, no que tange o stress e o rendimento escolar.

\section{REFERÊNCIAS}

1. Lemes SO, Fisberg MR, Gláucia M, Ferrini LG, Martins G, Siviero K, Ataka MA Stress Infantil e Desempenho Escolar: avaliação de crianças de 1a a 4a $^{\text {a }}$ série de uma escola pública do município de São Paulo. Estud. psicol. (Campinas). 2003; 2091): 5-14. doi: http://dx.doi.org/10.1590/S0103-166X2003000100001.

2. Camelo SHH, Angerami ELS. Sintomas de estresse nos trabalhadores atuantes em cinco núcleos de saúde da família. Rev. Latino-Am. Enfermagem. 2004 jan.fev; 12(1):14-21. doi: http://dx.doi.org/10.1590/S0104-11692004000100003.

3. Goulart E Jr, Lipp MEN. Estresse entre professoras do ensino fundamental de escolas públicas estaduais. Psicol Estud. 2008 out-dez; 13(4): 847-857. doi: http://dx.doi.org/10.1590/S1413-73722008000400023.

4. Lipp MEN. Prefácio. Nível de estresse auto percebido. In M. E. N. Lipp (Org.) O stress no Brasil: pesquisas avançadas. Campinas: Papirus; 2004. p. 11-13.
5. Ursin H, Olff M. The stress response. In: Stanford S, Clare-Salmon P. Stress: from synapse to syndrome. San Diego: Academic Press;1993. p. 08-10.

6. Lipp MN, Souza EAPS, Romano ASF, Covolan MA. Como enfrentar o stress infantil. São Paulo: Icone; 1991.

7. Kazmier LJ. Estatística aplicada a economia e administração. Porto Alegre: McGraw-Hill; 1982. 375 p.

8. Lipp MEN, Lucarelli MD. Escala de stress infantil: ESI. São Paulo: Casa do Psicólogo; 1998.

9. Lipp MEN, Malagris LN. Manejo de estresse. In: B. Rangé. Psicoterapia comportamental e cognitiva: Transtorno psiquiátricos. Campinas: Livro Pleno;1999. p.279-293. 
10. Causey DL, Dubow EF. Development of a self-report measure for elementary school children, J Clin Child Psychol. 1992; 21(1): 47-59. doi: http://dx.doi. org/10.1207/s15374424jccp2101_8.

11. Compas BE, Malcarne VL, Fondacaro KM. Coping with stressful events in older children and young adolescents, J Consult Clin Psychol. 1988 Jun; 56(3): 405-411.

12. Lazarus RS, Folkman S. Stress, apraisal and coping. New York: Springer Publishing Company; 1984.

13. Lipp MEN, Arantes JP, Buritl, MS, Witzig T. O estresse em escolares. Psicol. Esc. Educ. 2002 Jun; 6(1): 51-56. doi: http://dx.doi.org/10.1590/S141385572002000100006

14. Vilela MV. Sintomas e fontes de stress em escolares de 1a a 4a séries. In Lipp MEN (Org.). Pesquisas sobre stress no Brasil. Campinas: Papirus; 1996. p. 255-274.
15. Tricoli VAC. Stress e aproveitamento escolar em crianças do 1 o grau[Dissertação]. Campinas (SP): PUC; 1997.

16. Pereira MIW. Dor abdominal e stress infantil[Dissertação]. Campinas (SP): PUC; 1996.

17. Rodrigues OMPR. et al. Desenvolvimento Cognitivo: o resultado do Inventário Portage Operacionalizado e a avaliação dos pais. XXXIV Reunião Anual de Psicologia, Ribeirão Preto, 2014. http://repositorio.unesp.br/ bitstream/handle/11449/116074/rodrigues_ompr_Id_bauru.pdf?sequence=1

18. Lipp MEN. Estresse e o turbilhão da raiva. São Paulo: Casa do Psicólogo; 2005.

19. Tricoli VAC. A criança e a escola. In: LIPP, M. N.P. (Org.). Crianças Estressadas: causas, sintomas e soluções. Campinas: Papirus; 2000. p. 123-148.

\section{Como citar este artigo/How to cite this article:}

Romanholo RA, Theodoro WWG, Baia FC, Pereira JE, Lucena ARN, Prestes J, Almeida FM. Estresse infantil e sua relação com o rendimento escolar da criança nas disciplinas de português e matemática J Health Biol Sci. 2017 Abr-Jun: 155-159. 